\title{
THE USE OF ALTERNATIVE EXPERIMENTAL MODELS ON MICROSURGERY TECHNIQUE'S TRAINING FOR MEDICINE UNDERGRADUTE STUDENTS
}

\author{
HERRMANN, Fábio ${ }^{1 *}$; PESENATTO, Gustavo Guthmann²; GOLDANI, Eduardo³; SILVA, \\ Jefferson Braga ${ }^{4}$. \\ 1,2 Pontifícia Universidade Católica do Rio Grande do Sul, Faculdade de Medicina, Graduação, Av. Ipiranga, \\ 6690, CEP: 90610-000 - Prédio 60 - Porto Alegre-RS, Brasil. \\ ${ }^{3}$ Pontifícia Universidade Católica do Rio Grande do Sul, Faculdade de Medicina, Laboratório de Habilidades \\ Médicas e Pesquisa Cirúrgica, Av. Ipiranga, 6690, CEP: 90610-000 - Prédio 64 - Porto Alegre-RS, Brasil. \\ ${ }^{4}$ Pontifícia Universidade Católica do Rio Grande do Sul, Faculdade de Medicina, Diretor, Av. Ipiranga, 6690, \\ CEP: 90610-000 - Prédio 60 - Porto Alegre-RS, Brasil. \\ ${ }^{*}$ Autor correspondente \\ e-mail: fabioherrmannmed@gmail.com
}

Received 12 November 2016; received in revised form 9 January 2017; accepted 14 January 2017

\section{RESUMO}

No Brasil, há grande carência de microcirurgiões devido ao fato de o treinamento ser longo, caro e exigir muita dedicação. Com o surgimento dos comitês de ética em pesquisa e para o uso de animais, torna-se cada vez mais restrito o treinamento cirúrgico dos acadêmicos utilizando animais de pequeno e médio porte. $O$ objetivo deste trabalho foi relatar o treinamento de acadêmicos de Medicina da Pontifícia Universidade Católica do Rio Grande do Sul (PUCRS) em microcirurgia no reparo de lesões de nervos periféricos por meio da técnica de tubulização. O treinamento, com duração de 12 semanas, ocorreu em laboratório de pesquisa do HospitalEscola da PUCRS empregando modelo experimental comercial de baixo custo (sobrecoxas de frango resfriadas adquiridas no comércio local da cidade de Porto Alegre) e consistiu na execução de técnicas de incisão medialmente ao fêmur, dissecação das estruturas adjacentes e exposição do nervo ciático, secção do nervo ciático e tubulização por meio de sutura dos cotos proximal e distal nas extremidades do tubo de silicone. O treinamento propiciou uma evolução na destreza das técnicas treinadas assim como no tempo necessário para a sua realização demonstrando que pedaços de frango comercializados são um excelente modelo para o treinamento microcirúrgico.

Palavras-chave: educação médica, microcirurgia, modelos cirúrgicos, treinamento, técnicas.

\begin{abstract}
In Brazil, there is a great lack of micro-surgeons due to the fact that the training is long, expensive and requires a lot of dedication. With the outbreak of Committee on Animal Research and Ethics, it becomes increasingly restricted the surgical training of academics using small and medium-sized animals. The objective of this work was to report the training of medical academics of the Pontifical Catholic University of Rio Grande do Sul (PUCRS) in microsurgery in the repair of peripheral nerve lesions by means of the tubulization technique. The 12-week training was carried out in a research laboratory of the PUCRS Hospital-School, using a low-cost commercial experimental model (cooled chicken thigh purchased in the local market of the city of Porto Alegre) and consisted in the execution of incision techniques Medial to the femur, dissection of the adjacent structures and exposure of the sciatic nerve, sciatic nerve section and tubulization by suturing the proximal and distal nerve at the ends of the silicone tube. The training provided an evolution in the skill of the trained technicians as
\end{abstract}


well as in the time necessary for its accomplishment demonstrating that commercialized chicken pieces are an excellent model for the microsurgical training.

Keywords: medical education, microsurgery, surgical models, training, techniques.

\section{INTRODUÇÃO}

A evolução da medicina, nas últimas décadas, exige o aprimoramento do treinamento dos futuros profissionais, sendo pauta importante de discussão das escolas formadoras. As principais tentativas concretizadas atêm-se, sobretudo, a mudanças metodológicas ou pedagógicas, com a reestruturação dos currículos (Gomes e Rego, 2011). Em pesquisas científicas, além das aulas experimentais de técnica operatória, a utilização de animais representa um dos dilemas mais conflitantes no debate bioético (Damy et al., 2010). Com o surgimento dos órgãos protetores dos animais, assim como dos comitês de ética em pesquisa e para o uso de animais, torna-se cada vez mais restrito o treinamento cirúrgico dos acadêmicos utilizando animais de pequeno e médio porte. No Brasil, existe grande carência de microcirurgiões devido aos custos envolvidos no treinamento e na escassez de serviços que ofereçam treinamento especializado. $\mathrm{O}$ treinamento em microcirurgia geralmente é longo, caro e exige alto grau de dedicação (Junior et al., 2014).

O uso racional de modelos animais em pesquisas biomédicas é necessário enquanto não forem desenvolvidos modelos e sistemas alternativos que possam se igualar aos modelos animais. A meta primordial do uso de modelos animais é auxiliar a solucionar problemas na prática clínica, contribuindo com o desenvolvimento de novos métodos na busca da cura e tratamento de doenças. Na área cirúrgica, a prática das técnicas é indicativo de bons resultados, condição que aumenta a importância do acadêmico aprimorar suas habilidades 0 precocemente, para se adequar ao ambiente cirúrgico. (Kinshoku et al, 2012).

Os avanços em pesquisa e ensino na área de microcirurgia permitiram a extensa aplicação desta técnica em diversas reconstruções, sendo possível utilizá-la em inúmeras áreas da cirurgia e outras especialidades, como Ortopedia e Oftalmologia. O domínio da técnica microcirúrgica é um processo longo e difícil, que requer grande habilidade manual, paciência, dedicação e treinamento contínuo. Em um laboratório de microcirurgia, pode-se aprender a operar o microscópio, familiarizar-se com o campo bidimensional e com os instrumentos, e os princípios da técnica microcirúrgica, sendo indispensável na obtenção dos resultados desejados. Entretanto, um dos maiores obstáculos na organização de um centro de treinamento apropriado é o custo. Tanto os instrumentos quanto os equipamentos ópticos adequados ao treinamento são de custos elevados. Assim, a busca por novos modelos cuja barreira econômica seja menor e cujos resultados sejam satisfatórios é muito estimulada pelo âmbito acadêmico. (Dias et al, 2010)

O modelo para treinamento experimental com patas de porco é um dos mais utilizado nos programas de residência médica em dermatologia nos Estados Unidos. O modelo simula a pele humana com custo reduzido, para ensino prático das operações sobre a pele e anexos. Essa metodologia tem uma boa receptividade por parte dos alunos e docentes, por fornecer uma boa introdução aos procedimentos, e o estudante pode ser avaliado quanto às suas habilidades ao final do treinamento. Os resultados obtidos permitiram inferir que oficinas com esse modelo tiveram um papel fundamental no treinamento prático das habilidades cirúrgicas. (Purim et al, 2013)

A transecção de um nervo periférico resulta na perda de função do órgão alvo, que raramente pode ser revertido sem uma intervenção cirúrgica. Uma alternativa de tratamento de reparo para o nervo é a tubulização neural, que tem sido muito utilizada em estudos e está possibilitando o aumento do número de fibras nervosas regeneradas entre os cotos do nervo seccionado. (Oliveira et al, 2004) Para a melhor realização dessa técnica, a microcirurgia se faz necessária, e seu 
treinamento pode ser realizado em centros acadêmicos, com a utilização de tubos de silicone em modelos animais. $\mathrm{O}$ uso de modelos animais de pequeno porte também se mostrou indispensável para a pesquisa e desenvolvimento de técnicas em microcirurgia. Contudo, a sua alta capacidade regenerativa pode confundir a interpretação dos resultados, principalmente em relação ao sistema nervoso de roedores (Brenner, 2008).

No Brasil, as diretrizes curriculares da graduação recomendam formar médico generalista capaz de realizar procedimentos clínicos e cirúrgicos iniciais de emergência, urgência e de atendimento ambulatorial básico. Diante da extrema relevância das técnicas operatórias para a prática médica, o desenvolvimento de técnicas microcirúrgicas depende de diversos fatores como econômicos, éticos, didáticos e relevância para prática médica. Colocar o estudante no cenário de atividades práticas da disciplina para mobilizar conhecimentos, articular habilidades e atitudes diante dos objetivos propostos deve ser preconizada pelas instituições acadêmicas. (Purim, 2015) O aperfeiçoamento dos modelos experimentais pode alcançar, pelo corpo docente e discente, novas descobertas e a construção de conhecimentos mais significativos no ensino superior.

Este trabalho teve como objetivo relatar o treinamento em microcirurgia no reparo de lesões de nervos periféricos através da técnica de tubulização de acadêmicos de Medicina da Pontifícia Universidade Católica do Rio Grande do Sul (PUCRS) empregando um modelo experimental comercial de baixo custo.

\section{MATERIAIS E MÉTODOS}

Os treinamentos semanais, com duração de 2 horas cada sessão, foram realizados no Laboratório de Habilidades Médicas e Pesquisa Cirúrgica (LHMPC) da Faculdade de Medicina da PUCRS por 12 semanas. Para o treinamento, foram utilizadas sobrecoxas de frango resfriadas Ave Serra Granja Pinheiros LTDA (Figura 1) adquiridas em um mercado tradicional da cidade de Porto Alegre. As sobrecoxas estavam embaladas em bandejas com aproximadamente $700 \mathrm{~g}$ e 4 pedaços, ou seja, os acadêmicos não tiveram nenhum contato com o animal vivo.

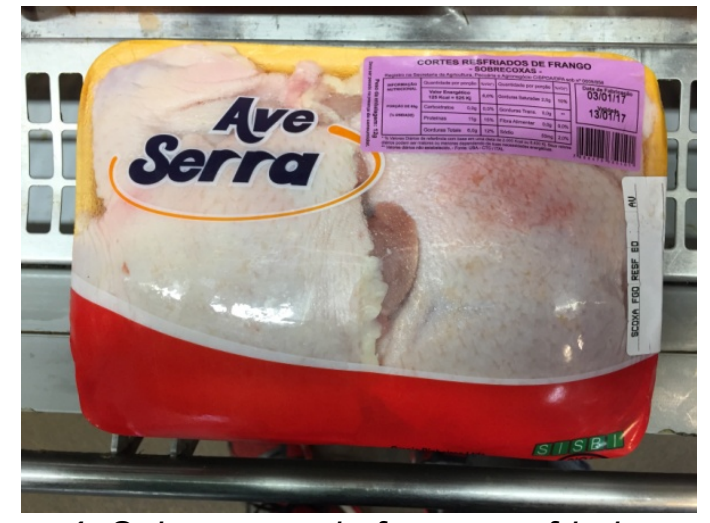

Figura 1. Sobrecoxas de frango resfriadas

Os alunos foram supervisionados e auxiliados por um monitor durante todo o treinamento. $O$ treinamento consistiu na exploração de técnicas de incisão medialmente ao fêmur, dissecação das estruturas adjacentes e exposição do nervo ciático, secção do nervo ciático, tubulização através de sutura do epineuro de cada coto neural (proximal e distal) nas extremidades do tubo de silicone com dois pontos simples em lados opostos do tubo finalizando com a introdução de $2,5 \mathrm{~mm}$ de cada coto neural no interior do tubo. Para a realização do treinamento, foram utilizadas pinças para microcirurgia (com dente e reta); tesoura curva e reta para microcirurgia; porta-agulhas para microcirurgia; fio de sutura de náilon monofilamentar 7,0 (Prolene $\AA$, BV 1, Ethicon $®$ ); microscópio de dissecção microcirúrgica (D.F.Vasconcellos, São Paulo, Brasil); tubo de silicone comercial de $15 \mathrm{~mm}$ de comprimento e 2 $\mathrm{mm}$ de diâmetro obtidos a partir de um segmento de uma sonda de aspiração traqueal $n^{\circ} 8$ da Mark Med®; e luvas de procedimento (Figuras 2 e 3 ).

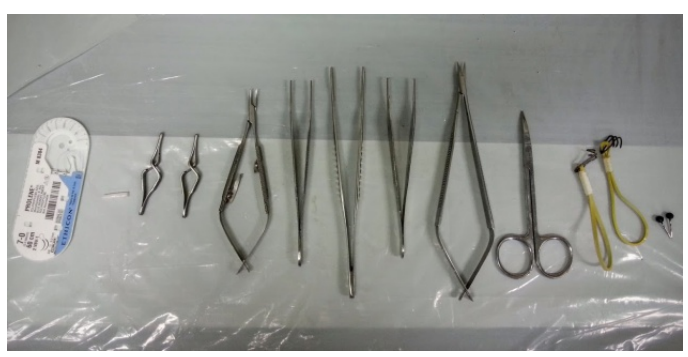

Figura 2. Instrumental empregado no 


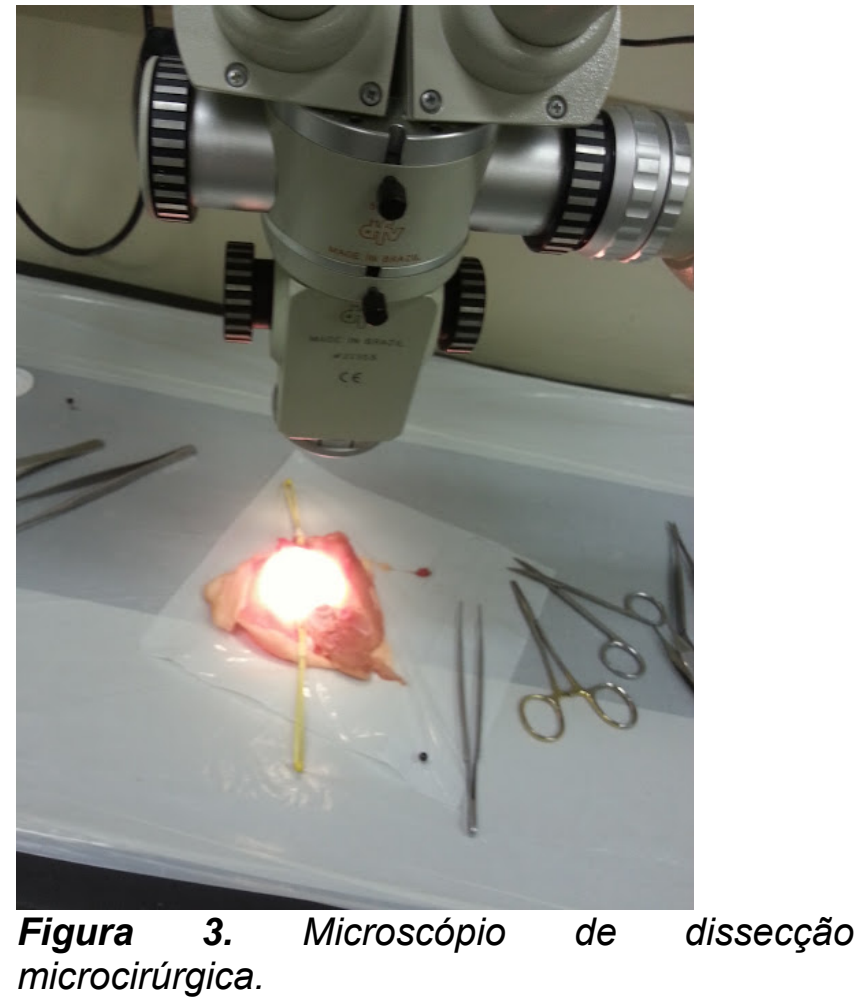

\section{RESULTADOS E DISCUSSÃO:}

Verificou-se, com o passar das sessões, uma evolução na destreza das técnicas treinadas assim como ao tempo necessário para a realização das técnicas. Nas duas primeiras semanas, onde foi treinada a dissecção das coxas (incisão medialmente ao fêmur e dissecação das estruturas), houve grande dificuldade na dissecção das estruturas e esse procedimento teve duração aproximada de $1 \mathrm{~h}$. Após quatro semanas de treinamento, esse procedimento passou a ser realizado em 30 minutos sem qualquer dano às estruturas subjacentes. Já na quinta e sexta semanas, onde foi realizado o treinamento de exposição e dissecção do nervo, as dificuldades encontradas relacionavam-se à dissecção das estruturas sem dividir os fascículos, secção indevida do nervo ciático e introdução e sutura do nervo em tubo de silicone. Foi necessário pouco mais de $1 \mathrm{~h}$ para o término desse procedimento que, nas duas semanas subsequentes, foi realizado em $45 \mathrm{~min}$. Após adquirir as primeiras habilidades, deflagrouse o treinamento de secção do nervo e introdução em tubo de silicone, suturando-se o epineuro de cada coto neural nas extremidades do tubo. $O$ processo completo inicialmente durava cerca de $1 \mathrm{~h} 30 \mathrm{~min}$ e, frequentemente, a sutura não ficava boa o suficiente para manter os segmentos do nervo firmemente dentro do tubo de silicone; após 4 semanas de treinamento, passou a ser realizado em 30 minutos em com excelente técnica de sutura mantendo os cotos proximal e distal do nervo firmemente suturados ao tubo. A cada treinamento, as técnicas foram aperfeiçoadas, aprimorando os resultados quanto a melhor dissecção, introdução dos cotos neurais, sutura e menor lesão neural (Figura 4 e Tabela 1).

Tabela 1. Evolução da técnica microcirúrgica.

\begin{tabular}{c|c|c}
\hline \multicolumn{3}{c}{ ALUNO 1 } \\
\hline Data & Tempo & $\begin{array}{c}\text { Introdução Cotos } \\
\text { Neurais }\end{array}$ \\
\hline $01 / 04 / 2016$ & $70 \mathrm{~min}$ & Não realizado \\
\hline $08 / 04 / 2016$ & $50 \mathrm{~min}$ & Não realizado \\
\hline $15 / 04 / 2016$ & $75 \mathrm{~min}$ & $1 \mathrm{~mm}$ e $1 \mathrm{~mm}$ \\
\hline $22 / 04 / 2016$ & $65 \mathrm{~min}$ & $1 \mathrm{~mm}$ e $1 \mathrm{~mm}$ \\
\hline $29 / 04 / 2016$ & $70 \mathrm{~min}$ & $2 \mathrm{~mm}$ e $2 \mathrm{~mm}$ \\
\hline $09 / 05 / 2016$ & $50 \mathrm{~min}$ & $2,5 \mathrm{~mm} \mathrm{e} 2 \mathrm{~mm}$ \\
\hline $13 / 05 / 2016$ & $45 \mathrm{~min}$ & $2 \mathrm{~mm}$ e $2 \mathrm{~mm}$ \\
\hline $20 / 05 / 2016$ & $55 \mathrm{~min}$ & $3,5 \mathrm{~mm}$ e $2,5 \mathrm{~mm}$ \\
\hline $27 / 05 / 2016$ & $55 \mathrm{~min}$ & $3 \mathrm{~mm}$ e $2,5 \mathrm{~mm}$ \\
\hline $03 / 06 / 2016$ & $45 \mathrm{~min}$ & $2,5 \mathrm{~mm}$ e $2,5 \mathrm{~mm}$ \\
\hline $10 / 06 / 2016$ & $40 \mathrm{~min}$ & $2,5 \mathrm{~mm}$ e $2,5 \mathrm{~mm}$ \\
\hline
\end{tabular}

\begin{tabular}{c|c|c}
\hline \multicolumn{3}{|c}{ ALUNO 2 } \\
\hline Data & Tempo & $\begin{array}{c}\text { Introdução Cotos } \\
\text { Neurais }\end{array}$ \\
\hline $01 / 04 / 2016$ & $75 \mathrm{~min}$ & Não realizado \\
\hline $08 / 04 / 2016$ & $60 \mathrm{~min}$ & Não realizado \\
\hline $15 / 04 / 2016$ & $70 \mathrm{~min}$ & $1 \mathrm{~mm}$ e $1 \mathrm{~mm}$ \\
\hline $22 / 04 / 2016$ & $70 \mathrm{~min}$ & $1 \mathrm{~mm}$ e $1 \mathrm{~mm}$ \\
\hline $29 / 04 / 2016$ & $70 \mathrm{~min}$ & $2 \mathrm{~mm}$ e $1,5 \mathrm{~mm}$ \\
\hline $09 / 05 / 2016$ & $55 \mathrm{~min}$ & $3 \mathrm{~mm}$ e $3 \mathrm{~mm}$ \\
\hline $13 / 05 / 2016$ & $45 \mathrm{~min}$ & $3 \mathrm{~mm}$ e $2,5 \mathrm{~mm}$ \\
\hline $20 / 05 / 2016$ & $50 \mathrm{~min}$ & $2 \mathrm{~mm}$ e $2 \mathrm{~mm}$ \\
\hline $27 / 05 / 2016$ & $45 \mathrm{~min}$ & $3 \mathrm{~mm}$ e $3 \mathrm{~mm}$ \\
\hline $03 / 06 / 2016$ & $45 \mathrm{~min}$ & $2 \mathrm{~mm}$ e $2,5 \mathrm{~mm}$ \\
\hline $10 / 06 / 2016$ & $40 \mathrm{~min}$ & $2,5 \mathrm{~mm}$ e $2,5 \mathrm{~mm}$ \\
\hline
\end{tabular}

\section{CONCLUSÕES:}

Com o objetivo de contribuir com a aquisição de habilidades cirúrgicas pelos alunos de graduação do Curso Medicina e, por 
consequência, beneficiar as pesquisas em cirurgia experimental, este trabalho traz à tona técnicas para auxiliar nos treinamentos em microcirurgia, além de aspectos éticos e educacionais referentes à cirurgia experimental. Por questões éticas, todo procedimento realizado em animais deve ser feito por profissionais habilitados ${ }^{4}$. Logo, a fim de evitar sacrifício dos animais de experimentação, é indispensável uma prévia formação teórico-prática em modelos experimentais comerciais de baixo custo para adquirir experiências fundamentais para a posterior realização de técnicas cirúrgicas nesses animais in vivo. A utilização de pedaços de frango adquiridos comercialmente é um excelente modelo para $\mathrm{O}$ treinamento microcirúrgico, pois, além de simular um modelo real, poupa o sacrifício de modelos animais para a fase inicial de aprendizado acadêmico em microcirurgia. Baseando-se em uma rotina preestabelecida e bem definida, na supervisão qualificada e na utilização de materiais de baixo custo, o treinamento microcirúrgico realizado mostrou-se satisfatório para 0 aprendizado acadêmico. Além disso, em 12 semanas, a prática com a instrumentação e os resultados microcirúrgicos apontaram uma evolução notável entre os participantes. Desse modo, o treinamento de técnicas de microcirurgia com este modelo experimental proporciona ao acadêmico a aquisição de conhecimento prático e de habilidades satisfatórios para realização de procedimentos iniciais que facilitarão na futura prática cirúrgica profissional.

AGRADECIMENTOS: À Faculdade de Medicina da PUCRS pela infraestrutura, materiais e equipamentos disponibilizados.

\section{REFERÊNCIAS:}

1. Gomes, A.P., Rego, S. Transformação da Educação Médica: É Possível Formar um Novo Médico a partir de Mudanças no Método de Ensino-Aprendizagem? Revista brasileira de educação médica, 2011, 35(4):557-566.
2. Damy, S. B., Camargo, R. S., Chammas, R., Figueiredo. L. F. P de. Aspectos fundamentais da experimentação animal aplicações em cirurgia experimental. Rev Assoc Med Bras, 2010; 56(1):103-11.

3. Junior, V. M., Silva, A. B. D. da., Groth, A. K., Lopes, M. A. C., Kurogi, A. S., Freitas, R. S., Tomasich, F. D. S. Modelo experimental alternativo para treinamento em microcirurgia. Rev. Col. Bras. Cir. 2014; 41(1):072-074.

4. Kinshoku, M. R, Rodriguez, C. A. L., Fidalgo, R. S., Duran, C. C. G., Leme, P. L. S., Duarte, I. S. Rational use of animal models for research and microsurgery training. Rev. Col. Bras. Cir., Rio de Janeiro, v. 39 , n. 5 , p. 414-417, Oct. 2012.

5. DIAS, lana Silva et al . Treinamento inicial em microcirurgia. Rev. Bras. Cir. Plást. (Impr.), São Paulo, v. 25, n. 4, p. 595599, Dec. 2010.

6. PURIM, Kátia Sheylla Malta et al. Avaliação de treinamento cirúrgico na graduação de medicina. Rev. Col. Bras. Cir., Rio de Janeiro, v. 40, n. 2, p. 152156, Apr. 2013.

7. Oliveira, A. L. R., Pierucci, A., Pereira, K. B. Peripheral Nerve Regeneration Through The Nerve Tubulization Technique. Instituto de Biologia, Universidade Estadual de Campinas, Braz. J. morphol. Sci. (2004) 21(4), 225231.

8. Brenner, M. J., Moradzadeh, A., Myckatyn, T. M., Tung, T. H. H., Mendez, A. B., Hunter, D. A. and Mackinnon, S. E. (2008), Role of timing in assessment of nerve regeneration. Microsurgery, 28: 265-272. doi:10.1002/micr.20483.

9. PURIM, Kátia Sheylla Malta; SKINOVSKY, James; FERNANDES, Júlio Wilson. Basic skills for outpatient surgery in medical graduation. Rev. Col. Bras. Cir., Rio de Janeiro, v. 42, n. 5, p. 341344, Oct. 2015. 

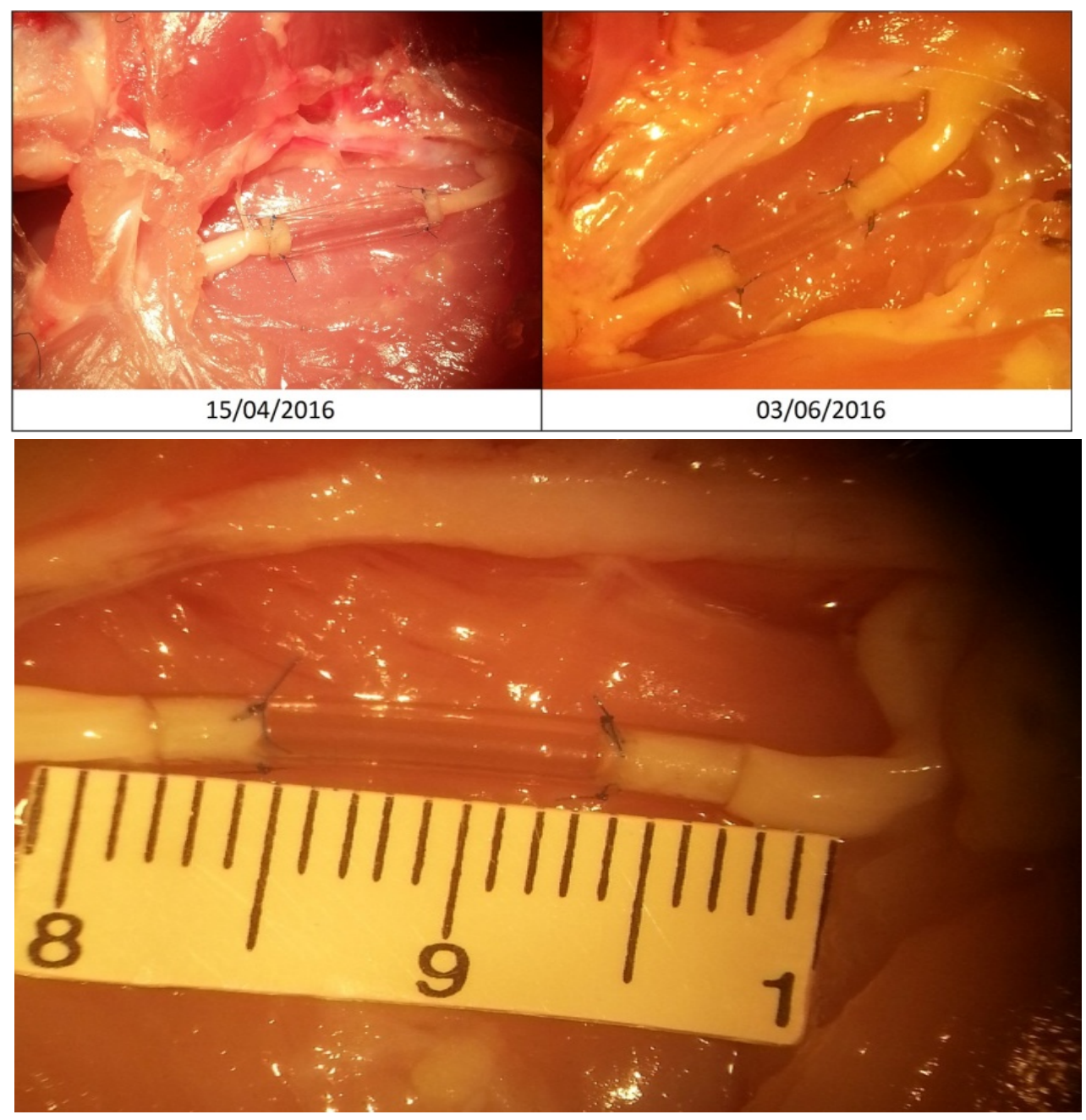

Figura 4. Evolução da técnica microcirúrgica de tubulização em nervos empregando modelos experimentais alternativos.

PERIÓDICO TCHÊ QUÍMICA • www.periodico.tchequimica.com • Vol. 14 N. 27 • ISSN 1806-0374 (impresso) • ISSN 1806-9827 (CD-ROM) • ISSN 2179-0302 (meio eletrônico)

(C) 2017. Porto Alegre, RS. Brasil

The Periódico Tchê Quimica (ISSN: 1806-0374; 2179-0302) is an open-access journal since 2004. Journal DOI: 10.52571/PTQ. http://www.tchequimica.com This text was introduced in this file in 2021 for compliance reasons.

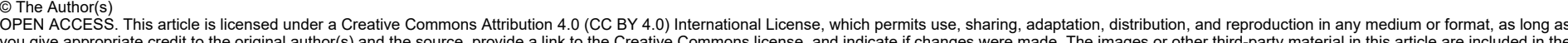
you give approps. or exceeds the permitted use, you will need to obtain permission directly from the copyright holder. To view a copy of this license, visit http://creativecommons.org/licenses/by/4.0/. 